\title{
Numerical simulation of the coupling between thermal dissipation and fish-eye crack growth in very high cycle fatigue regime
}

\author{
H.Q. NGUYEN, L. GALLIMARD and C. BATHIAS
}

Laboratoire Energ'etique, M'ecanique, Electromagn'etisme, Universite' Paris Ouest Nanterre-La D'éfense, 50 rue de S'evres - 92410 Ville d'Avray, France

ABSTRACT In this paper, we study the temperature field associated with the propagation of a fatigue crack in a very high cycle fatigue regime during ultrasonic fatigue testing. We use a Paris-Hertzberg crack growth law to compute the evolution of the crack and a perfectly elastic-plastic constitutive law to compute the plastic dissipation per cycle at the tip of the crack. A thermomechanical finite element model is proposed to estimate the evolution of the temperature field during the crack propagation. Numerical results obtained agree fairly well with experimental results.

Keywords fatigue crack growth; finite element model; fish-eye fracture; plasticity; thermomechanical analysis; ultrasonic fatigue; very high cycle fatigue.

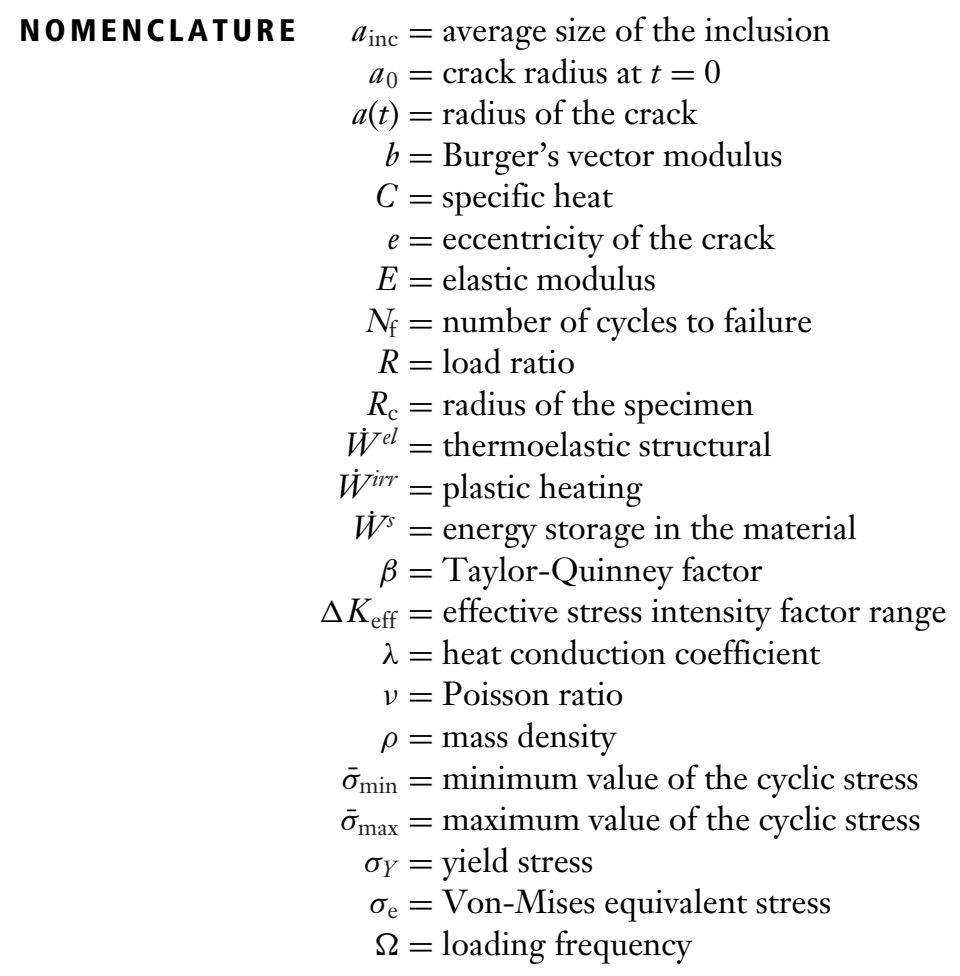

\section{INTRODUCTION}

The considerable attention given, in the past ten years, to fatigue fracture of metallic material in very high cycle regime, is mainly due to the increasing use of engineering materials in applications with service lives reach-

Correspondence: L. Gallimard. E-mail: laurent.gallimard@u-paris10.fr ing up to $10^{10}$ load cycles. It has been shown that very high cycles fatigue (VHCF) failures of steels are characterized by a fracture which does not occurs on the surface but rather internally in the material, especially in high strength steels. ${ }^{1-3}$ The initiation of fatigue cracks can be considered differently from physical or mechanical points of view. At the microscopic level, Mughrabi shows that the initiation of fatigue crack in the gigacycle fatigue regime 
can be described in terms of a micro structurally irreversible portion of the cumulative cycle strain. This means that there is no basic difference between fatigue mechanisms in low, mega, and gigacycle fatigue except for the strain localization. However, specific mechanisms can occur depending on the fatigue life. The fatigue life seems to be a key parameter to determine correctly the fatigue initiation location. In low cycle fatigue, in mega cycle fatigue and in gigacycle fatigue different mechanisms can operate at different scales of plasticity. In the low cycle fatigue regime, the cyclic plastic deformation is critical at the surface but exists also in the bulk of the metal. Typically, several cracks nucleate from the surface. When the fatigue life is below $10^{5}$ cycles, general plastic deformation of the specimen bulk governs the initiation. When the fatigue life is between $10^{6}$ and $10^{7}$ cycles the plastic deformation depends on the plane stress surface effect and the presence of flaws, which explain the critical location of fatigue initiation. Typically, the initiation starts with one crack only, from the surface. However approaching $10^{9}$ cycles the plastic deformation in plane stress conditions vanishes; the macroscopic behaviour of the metal is elastic except around flaws, metallurgical defects or inclusions. In very high cycle fatigue, the plane stress conditions are not enough for a surface plastic deformation according the Von Mises criteria. The initiation may be located in an internal zone. When the crack initiation site is in the interior, this leads to the formation of one fish eye on the fracture surface, typical of gigacycle fatigue. In this case, the cyclic plastic deformation is related to the stress concentration around a defect: inclusion, porosity, supergrain. Because the probability of occurrence of a flaw is greater within an internal volume than at a surface, the typical initiation in gigacycle fatigue will be most often in the bulk of the metal.

During VHCF, the cracks nucleate at microscopic flaws and defects, pore, inclusion, and the crack growth leads to the formation of a structure so-called fish-eye, which could be described as a circular pattern that develops on the fracture surfaces. Ultrasonic fatigue testing ${ }^{1,4,5}$ is a powerful tool for evaluating VHCF properties. It uses a high frequency of $20 \mathrm{kHz}$, which complete $10^{9}$ cycles in a day. The crack propagation in the tested specimen leads to a cyclic plastic dissipation and thus to a source of heat generation. One way of assessing this dissipated energy is to use temperature measurements because part of this dissipation will occur as heat. To this end infrared cameras can be used. ${ }^{6,7}$ As far we know few studies have been devoted to the computation of the temperature field associated with the propagation of a fatigue crack during VHCF failure. ${ }^{8,9}$

Our research group has proposed a first simplified analytical approach to model the thermal dissipation during the fish eye propagation, ${ }^{8}$ this modification assume that the thermal dissipation is distributed uniformly around the crack tip. The purpose of this work is to enhance this first approach by introducing a mechanical modelling of the cyclic plastic dissipation, without assuming a priori an uniform distribution. We propose a finite element based computational model of the crack propagation during a VHCF failure, in order to compute the temperature field evolution in the tested specimen. The crack propagation is modelled by a classical Paris-Hertzberg crack growth law, and the plastic dissipation per cycle is determined by 3D-elastic plastic finite element modelling of stationary mode I crack under constant amplitude loading. A fraction of this plastic dissipation is converted heat and used as a mobile thermal source to compute the temperature field evolution.

The paper is organized as follows: In the first section, the crack growth law and the thermomechanical model are developed. The next section is devoted to the finite element modelling. Finally, the last section the temperature field obtained by the proposed approach is compared with experimental data and the evolution of the plastic dissipation as well as the temperature field around the crack are studied.

\section{MODEL DESCRIPTION}

We consider a cylinder $C$ which has a circular crosssection of radius $R_{\mathrm{c}}$ and a height $2 L$. A small circular crack perpendicular to the cylinder axis lies in the centre of the cylinder, as shown in Fig. 1. The radius of the crack is denoted by $a(t)$ and its eccentricity from the

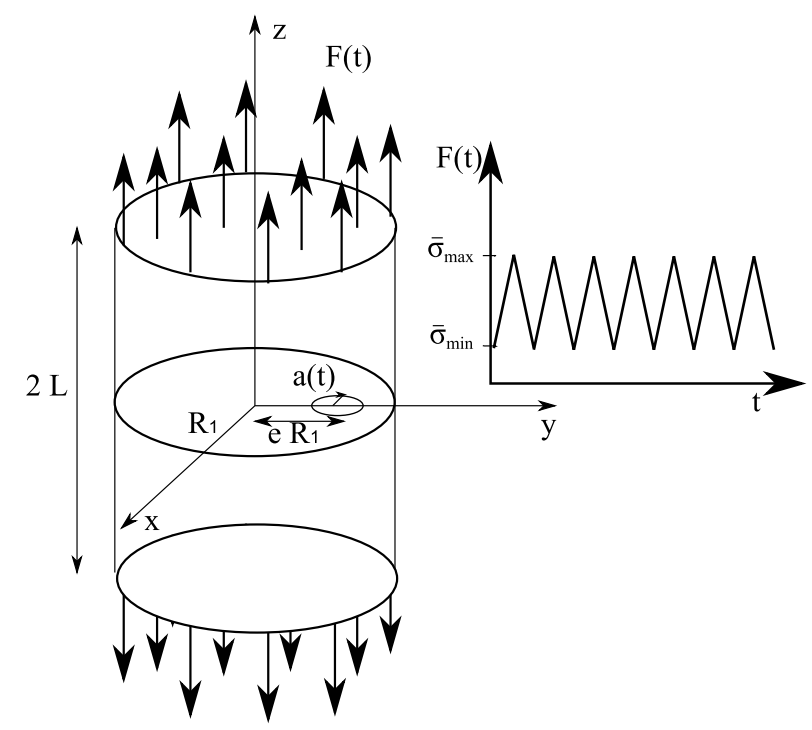

Fig. 1 Model description. 


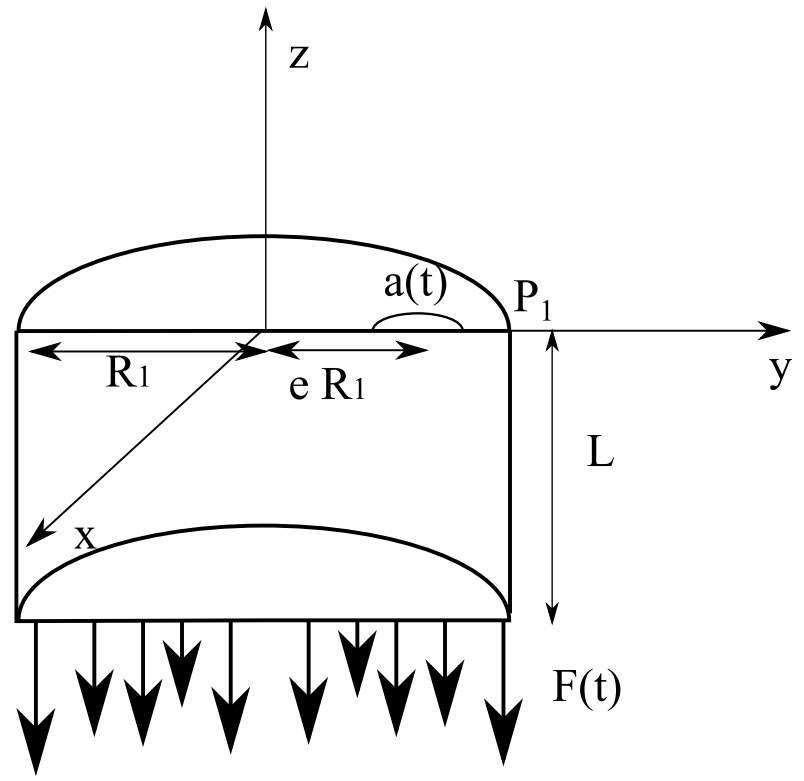

Fig. 2 Model description: one quarter of the cylinder.

centre of $C$ is denoted by $e$. $C$ is submitted to a cyclic loading: During each cycle the stress is varied linearly from an initial minimum value $\bar{\sigma}_{\min }$ to a maximum value $\bar{\sigma}_{\max }$ and back to the initial value $\bar{\sigma}_{\min }$. An important parameter used to characterize the cyclic load is the so-called load ratio, defined as $R=\bar{\sigma}_{\min } / \bar{\sigma}_{\max }$. Because of symmetry conditions only one quarter of the cylinder is modelled (Fig. 2).

\section{Crack growth model}

Paris and co-workers ${ }^{5}$ have developed estimation for crack growth life for internal initiation, termed fish-eye using the Paris-Hertzberg crack growth rate law. ${ }^{10}$ In the present work we use this law to describe the growth of the fatigue crack

$\frac{\mathrm{d} a}{\mathrm{~d} N}=b\left(\frac{\Delta K_{\mathrm{eff}}}{E \sqrt{b}}\right)^{3}$,

where $b$ is the Burger's vector modulus, $E$ the elastic modulus and $\Delta K_{\text {eff }}$ the effective stress intensity factor range. In this first approach we consider the following simplifications in the history of the crack propagation: the crack is supposed to remain circular during the crack growth process, the crack closure effect is neglected and we use the analytical formula for a circular crack in an infinite media. ${ }^{11}$

$\Delta K_{\mathrm{eff}}=\Delta K=\frac{2}{\pi} \Delta \sigma \sqrt{\pi a}$.
Let us denotes $a_{0}$ the crack radius at $t=0$, and by $\Omega$ the loading frequency. Hertzberg ${ }^{10}$ notes that the threshold corner is such that

$\frac{\mathrm{d} a}{\mathrm{~d} N}=b$ when $a=a_{0}$.

These assumptions lead to the following expression for the crack radius

$a(t)=\frac{a_{0}}{\left(1-\frac{b \Omega t}{2 a_{0}}\right)^{2}}$.

The crack grows until reaching the surface of the cylinder at $t=T_{\mathrm{f}}$ when $a\left(T_{\mathrm{f}}\right)=R_{\mathrm{c}}(1-e)$.

$$
T_{\mathrm{f}}=\frac{2 a_{0}}{b \Omega}\left(1-\sqrt{\frac{a_{0}}{R_{\mathrm{c}}(1-e)}}\right) .
$$

\section{Thermal model}

We consider a problem of a fatigue crack in an elasticplastic material. The temperature field in the cylinder is evaluated by solving the heat transfer equation

$\rho C \frac{\partial T}{\partial t}=\lambda \Delta T+\dot{W}^{e l}+\dot{W}^{i r r}-\dot{W}^{s}$

where $\rho$ is the mass density, $C$ the specific heat, $\lambda$ the heat conduction coefficient. $\dot{W}^{e l}, \dot{W}^{i r r}, \dot{W}^{s}$ are respectively, the thermoelastic structural heating, the plastic heating and the energy storage in the material through the creation of micro-stress fields linked to the development of dislocations. Under fatigue loading the thermoelastic source has the same frequency spectrum as the stress signal and the variation in thermoelatic energy $W^{e l}$ vanishes at the end of each loading cycle. ${ }^{12}$ As we are interested in the temperature variation over a great number of cycles, the temperature may be considered averaged over one complete cycle and the mean temperature rise in the material will be due to the inelastic effects only. Thus the thermoelastic heating $\dot{W}^{e l}$ can be taken out of Eq. (6). The energy storage term $\dot{W}^{s}$ is usually computed by considering that it represents between $5 \%$ and $15 \%$ of the plastic heating $\dot{W}^{\text {irr }} \cdot{ }^{13,14}$

The two can be merged in a unique expression written as $\beta \dot{W}^{i r r}$, where $\beta$ is a multiplicative factor, called the Taylor-Quinney factor, which classically is taken between 0.85 and 0.95 . Mean plastic energy dissipation per cycle is computed ${ }^{15}$

$\dot{W}^{i r r}=\Omega \frac{\mathrm{d} W^{i r r}}{\mathrm{~d} N}=\Omega\left[\int_{\text {cycle }} \sigma: \mathrm{d} \varepsilon^{p}\right]$. 
Plastic wake behind the crack tip

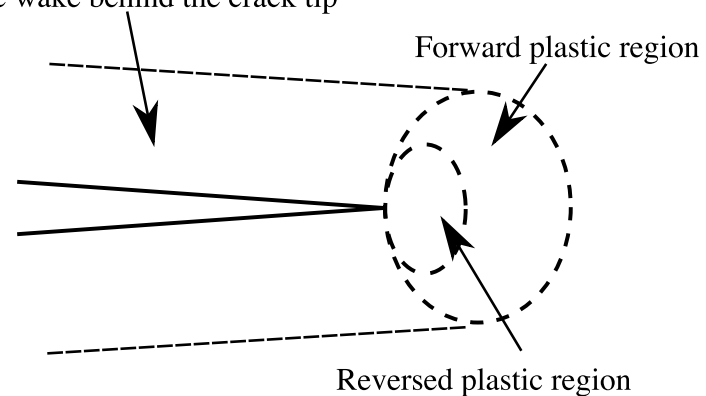

Fig. 3 Plastic regions during cyclic loading.

where $\Omega$ is the frequency of the loading, to obtain a simplified model of the heat transfer equation

$\rho C \frac{\partial T}{\partial t}=\lambda \Delta T+\beta \Omega\left[\int_{\text {cycle }} \sigma: \mathrm{d} \varepsilon^{p}\right]$.

It was shown that the characteristic time of the heat convection is higher than the crack propagation time and that the losses due to the heat convection could be neglected during the crack propagation. ${ }^{8}$ These considerations lead to the introduction of adiabatic conditions on the surface of the specimen. Moreover, symmetry conditions on the crack plane allow adiabatic condition to be chosen on that plane.

\section{Computation of the plastic energy dissipation}

The plastic energy dissipation per cycle is obtained by a 3D elastic-plastic finite element analysis. Three plastic regions exist during the cyclic loading, the forward plastic region which forms during the loading, the reverse plastic region which develops when the load is reversed and the plastic wake which develops when the crack extend in the plastic region (Fig. 3). It was shown that in VHCF regime, ${ }^{16}$ the crack growth is not a significant portion of life in gigacycle fatigue with fish-eye failure (less than 1\%). However, the number of cycles involved is still important $\left(\approx 10^{5}\right.$ cycles) and a direct nonlinear computation will lead to prohibitive computational time. In this respect, we propose to follow the approach developed by Klingbeil ${ }^{15}$ and to compute the energy dissipation per cycle $\mathrm{d} W^{i r r} / \mathrm{d} N$ during a single load cycle on a stationary crack for different radius $a_{i}\left(a_{i n}=a_{0}<a_{1}<\cdots<a_{n}=a_{f i}\right)$. To simulate the plastic fatigue regime, and not a monotonic loading, two cycles are computed for each radius $a_{i}$ and only the last cycle is used to evaluate the plastic dissipation. Such an approach neglects the contribution of the actual crack extension during any given load cycle. However, for Paris-Regime crack growth, both the plastic work and the surface energy contributions associated with the actual crack extension in any given cycle are negligible com- pared to the total plastic dissipation. In VHCF regime the imposed load is small compared to the yield strength and the contribution of the plastic wake will be neglected in our work. Finally an elastic-perfectly plastic constitutive model is considered in this work. The yield function is defined by

$f(\sigma)=\sigma_{e}-\sigma_{Y}$,

where $\sigma_{Y}$ is the yield stress and $\sigma_{e}$ is the Von-Mises equivalent stress defined from the deviatoric stress $\sigma^{D}$ by

$\sigma_{e}=\sqrt{\frac{3}{2} \sigma^{D}: \sigma^{D}}$.

During the cyclic loading, part of the crack surfaces, may be under contact and contact between surface is modelled by a unilateral contact law without friction.

\section{FINITE ELEMENT COMPUTATION}

The total plastic dissipation per cycle is obtained by a 3-D elastic-plastic finite element analysis using Cast $_{3} M^{17}$ a general FE computer code for the analysis of structures by the finite element method. This code was developed by the Mechanical Department and Technology (DMT) of the French Police station with Atomic Energy (ECA). The finite element computation is done in two steps. In a first step a set of mechanical computations are done for crack radius $a_{i}\left(a_{i n}=a_{0}<a_{1}<\cdots<a_{n}=a_{f i}\right)$. The energy dissipation in the structure during one cycle for each selected $a_{i}$ is denoted $\left(\mathrm{d} W^{i r r} / \mathrm{d} N\right)\left(a_{i}\right)$. Each of these computations consists in the analysis of two loading cycles. To obtain an accurate value of the energy dissipation at the crack tip we use linear 3D elements, with a sufficient number of elements in the reversed plastic region. ${ }^{18}$ Because of symmetry conditions only one quarter of the cylinder is meshed as shown in Fig. 4. Figure 5 shows the mesh in the plane of the crack for a fixed crack length and Fig. 6 is a zoom of the 3D mesh around the crack tip. The number of time step is set to $p$ for each cycle, a trapezoidal integration rule is used to estimate the value of the dissipation during one cycle on each Gauss point in the mesh.

$\frac{\mathrm{d} W^{i r r}}{\mathrm{~d} N}\left(a_{i}\right)=\int_{\text {cycle }} \sigma: \mathrm{d} \varepsilon^{p}=\sum_{j=1}^{p}\left(\sigma_{j+1}+\sigma_{j}\right):\left(\varepsilon_{j+1}^{p}-\varepsilon_{j}^{p}\right)$.

In a second step, the thermal problem is solved with an implicit integration scheme. The time discretization $\left(t_{0}<t_{1}<\cdots<t_{n}\right)$ is chosen such that $a\left(t_{i}\right)=a_{i}$ thanks to Eq. (4). Where $t_{0}=0$ designates the initiation of the fish-eye (i.e. $\left.a\left(t_{0}\right)=a_{0}\right)$ and $t_{n}=T_{\mathrm{f}}$ designates the instant when the crack reach the specimen surface as defined in Eq. (5). The loading is given by the energy dissipation 


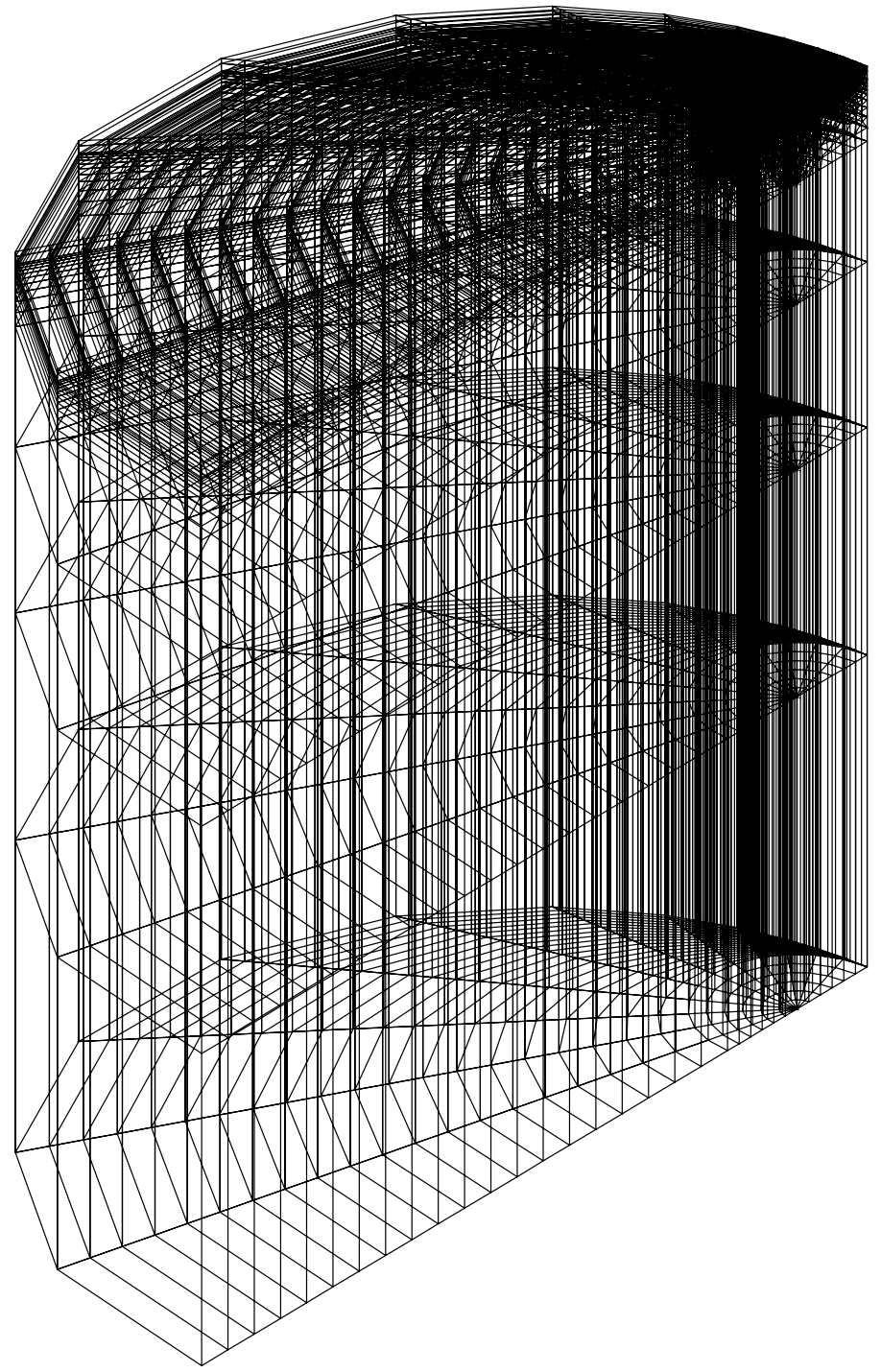

Fig. 4 Finite element mesh.

per cycle computed in Eq. (11). The meshes used are the meshes used during the mechanical computation to avoid errors due the data transfer on $\left(\mathrm{d} W^{i r r} / \mathrm{d} N\right)\left(a_{i}\right)$ between different meshes.

\section{NUMERICAL RESULTS}

\section{Comparison with measured data}

In this section the hypothesis chosen to build the numerical model are evaluated by a comparison with an experimental result. The tested material is a high-strength steel SAE 5120. Fatigue test are performed at ultrasonic fatigue frequency $\Omega=20 \mathrm{kHz}$ with a stress ratio of $R=0.1$ and stress amplitude $\Delta \sigma=400 \mathrm{MPa}$, using compressed air of $20{ }^{\circ} \mathrm{C}$ to cool the specimen. Both the ultrasonic fatigue machine and the specimen were described in previous pa- per. ${ }^{19,20}$ During the loading, an infrared camera is used to measure and record the temperature at the surface of the specimen. The acquisition frequency was $100 \mathrm{~Hz}$ with each image consisting of $64 \times 80$ pixels and a spatial resolution of $0.12 \mathrm{~mm} /$ pixel. Two tests have been conducted. In Table 1 are given the number of cycles to failure $N_{\mathrm{f}}$, the crack parameters measured after the failure $a_{\text {inc }}$ and $e$, as well as the radius of the crack at $t=0$ which is defined by $a_{0}=a_{\text {inc }} / 0.94$ as proposed by Paris. ${ }^{21}$ The final time

Table 1 Experimental parameters

\begin{tabular}{llllll}
\hline Test & $N_{\mathrm{f}}$ (cycles) & $a_{\text {inc }}(\mu \mathrm{m})$ & $e$ & $a_{0}(\mu \mathrm{m})$ & $T_{\mathrm{f}}(\mathrm{s})$ \\
\hline 1 & $5.25 \times 10^{7}$ & 10.03 & 0.79 & 10.67 & 4.84 \\
2 & $4.24 \times 10^{7}$ & 13.80 & 0.83 & 14.68 & 6.20 \\
\hline
\end{tabular}




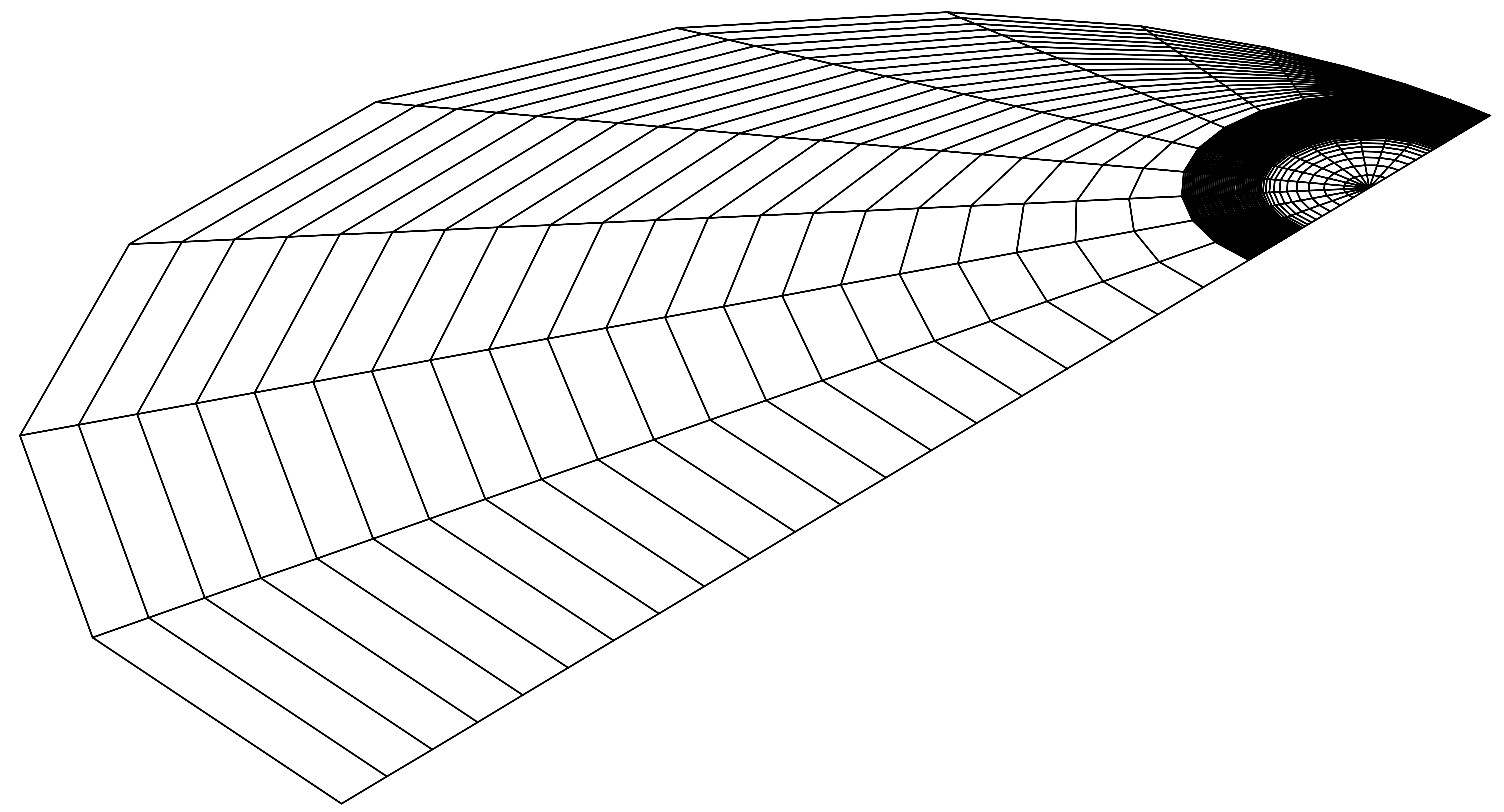

Fig. 5 Projection of the mesh in the crack plane.

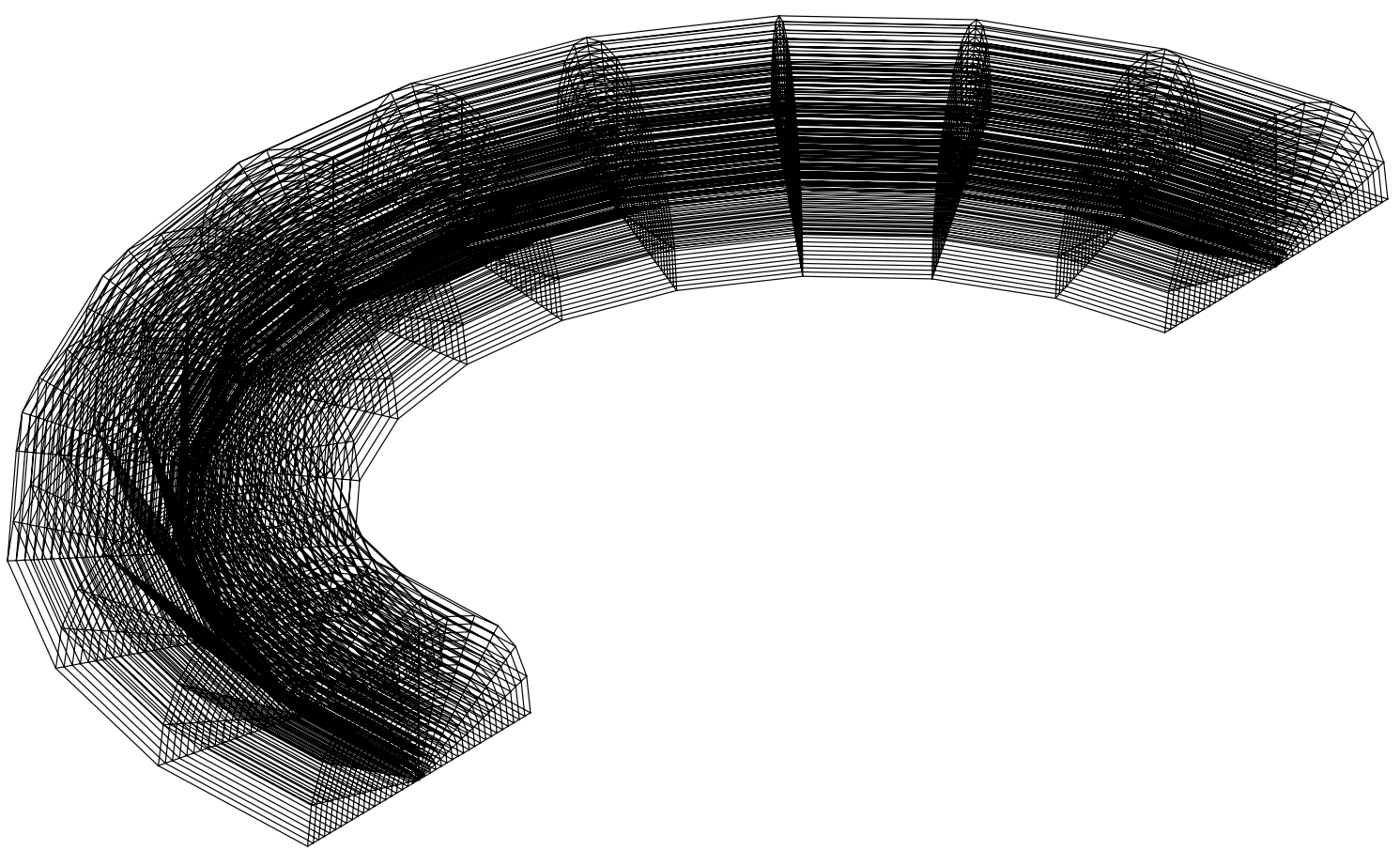

Fig. 6 Finite element mesh around the crack front.

step is computed from Eq. (5) where the Burger vector's modulus is taken such that $b=1.8 \times 10^{-10} \mathrm{~m}$ and the radius of the specimen is supposed to be $R_{\mathrm{c}}=1.5 \mathrm{~mm}$. The computations lead to $T_{\mathrm{f}}=4.84 \mathrm{~s}$ for test 1 and $T_{\mathrm{f}}=$ $6.20 \mathrm{~s}$ for test 2 . Figure 7 shows the evolution of the temperature measured on point $P_{1}\left(0, R_{1}, 0\right)$ (see Fig. 1) dur- ing the formation of the fish-eye (i.e. on the time interval $\left.\left[0, T_{\mathrm{f}}\right]\right)$. The measured temperature at the beginning of the initiation of the fish eye was $T(t=0)=21.9^{\circ} \mathrm{C}$ for test 1 and $T(t=0)=24^{\circ} \mathrm{C}$ for test 2 . It can be seen that the plastic work leads to a fast increase of the temperature. 


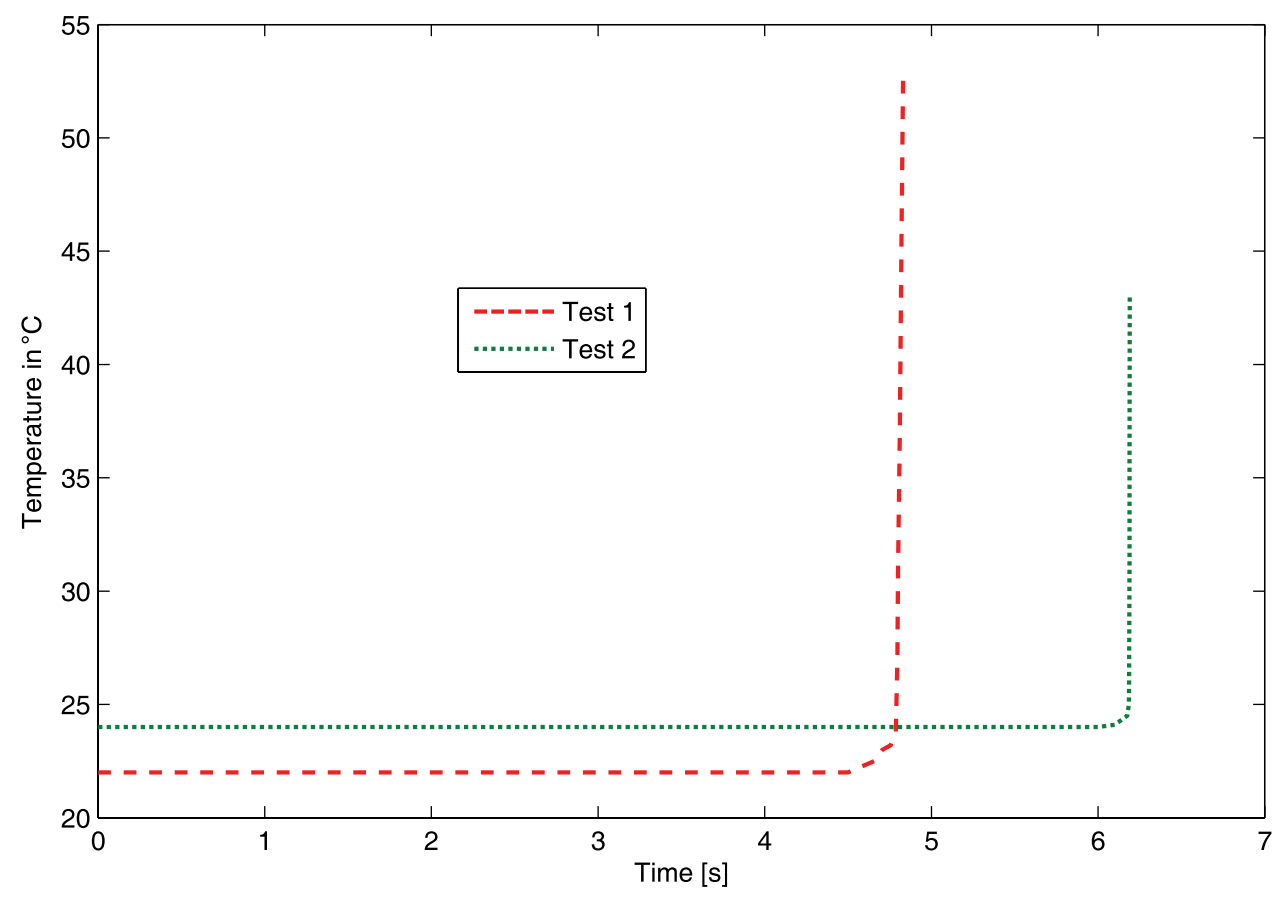

Fig. 7 Evolution of the temperature during the formation of the fish-eye for an SAE5120. Stress amplitude (400 MPa), load ratio (0.1) and testing frequency $(20 \mathrm{kHz})$.

The material is approximated as elastic-perfectly plastic with thermomechanical properties $E=200 \mathrm{GPa}$ (elastic modulus), $v=0.3$ (Poisson ratio), $\sigma_{Y}=1067 \mathrm{MPa}$ (yield stress), $\rho=7800 \mathrm{kgm}^{-3}$ (mass density), $C=$ $460 \mathrm{~J} \mathrm{~K}^{-1} \mathrm{~kg}^{-1}$ (specific heat), $\lambda=52 \mathrm{WK}^{-1} \mathrm{~m}^{-2}$ (heat conduction coefficient). The radius of the specimen is supposed to be $R_{\mathrm{c}}=1.5 \mathrm{~mm}$ and its height is defined by $L=6 \mathrm{~mm}$. The coupled thermomechanical problem defined in the preceding sections is used to compute the evolution of the temperature on the time interval $\left[0, T_{\mathrm{f}}\right]$, where $t=0$ denotes the crack initiation and $t=T_{\mathrm{f}} \mathrm{de}-$ notes the failure of the specimen. The initial temperature is assumed to be constant in the specimen and set to the measured data: $T(t=0)=21.9^{\circ} \mathrm{C}$ for test 1 and $T(t=0)=24^{\circ} \mathrm{C}$ for test 2 . The comparison between the measured and the computed temperature are plotted in Fig. 8 for test 1, and in Fig. 9 for test 2. These comparisons show a good agreement between the predicted and the measured values. In particular, we observe that, accordingly to the experimental results, the final temperature computed in test 1 is greater than the final temperature computed in test 2 . Numerically this fact is linked to the eccentricity of the crack. In test 1 the eccentricity which is smaller than in test 2 leads to a greater final radius of the crack, and thus to a greater plastic work and a greater temperature increase.

\section{Numerical study of the plastic dissipation and of the temperature rise}

The following results illustrate the evolution of the plastic dissipation per cycle, and of the temperature near the crack tip. The calculations are done with the geometric and materials values used for test 1 . The evolution of the plastic dissipation is computed as a function of the crack length. To study the evolution of the plastic dissipation around the crack, we have divided the crack tip line in regular angular sections $\left(0=\theta_{0}<\theta_{1}<\cdots<\theta_{N}=180\right)$, and the structure in $N$ elementary volumes $V\left(\left[\theta_{i-1} \theta_{i}\right]\right)$ as shown in Fig. 10. The plastic dissipation per cycle is computed in each volume $V\left(\left[\theta_{i-1} \theta_{i}\right]\right)$ as a function of the crack length.

$\frac{\mathrm{d} W^{i r r}}{\mathrm{~d} N}\left(\left[\theta_{i-1} \theta_{i}\right]\right)=\left(\int_{V\left(\left[\theta_{i-1} \theta_{i}\right]\right.}\left(\int_{c y c l e} \sigma: \mathrm{d} \varepsilon^{p}\right) \mathrm{d} V\right)$.

The evolution of the plastic dissipation for angular sections of $15^{\circ}$ is shown in Fig. 11. It is important to note that the plastic dissipation is not distributed regularly around the crack tip line as the crack progresses and is mainly concentrated in the two elementary volume which are the nearest to the surface.

The contours of the temperature field near the crack tip are shown in Figs 12-14 at different times during the crack 


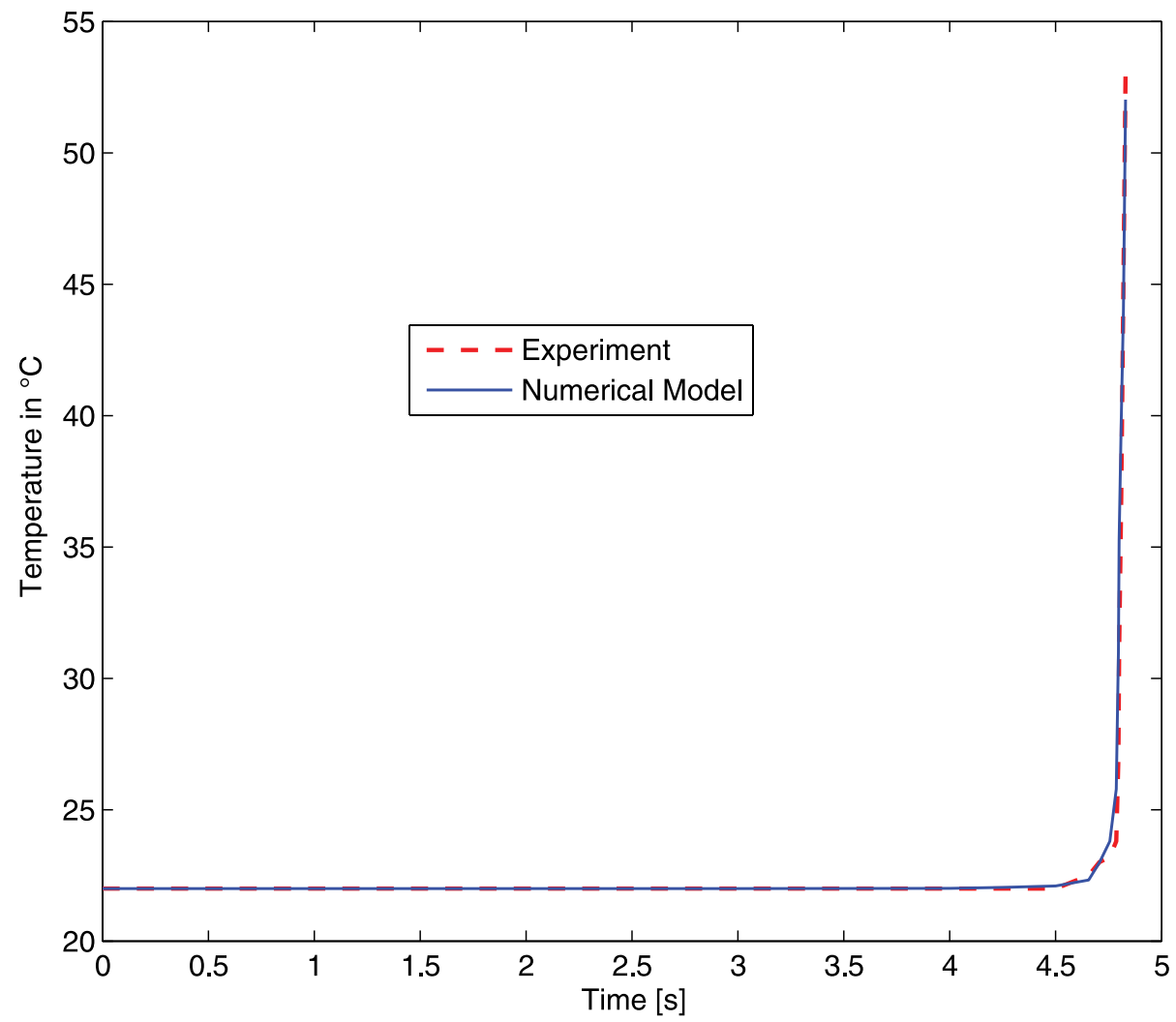

Fig. 8 Test 1: Comparison between the modeled and the experimental temperature during the formation of the fish-eye (initial inclusion $10.03 \mu \mathrm{m}$, eccentricity 0.79 ).

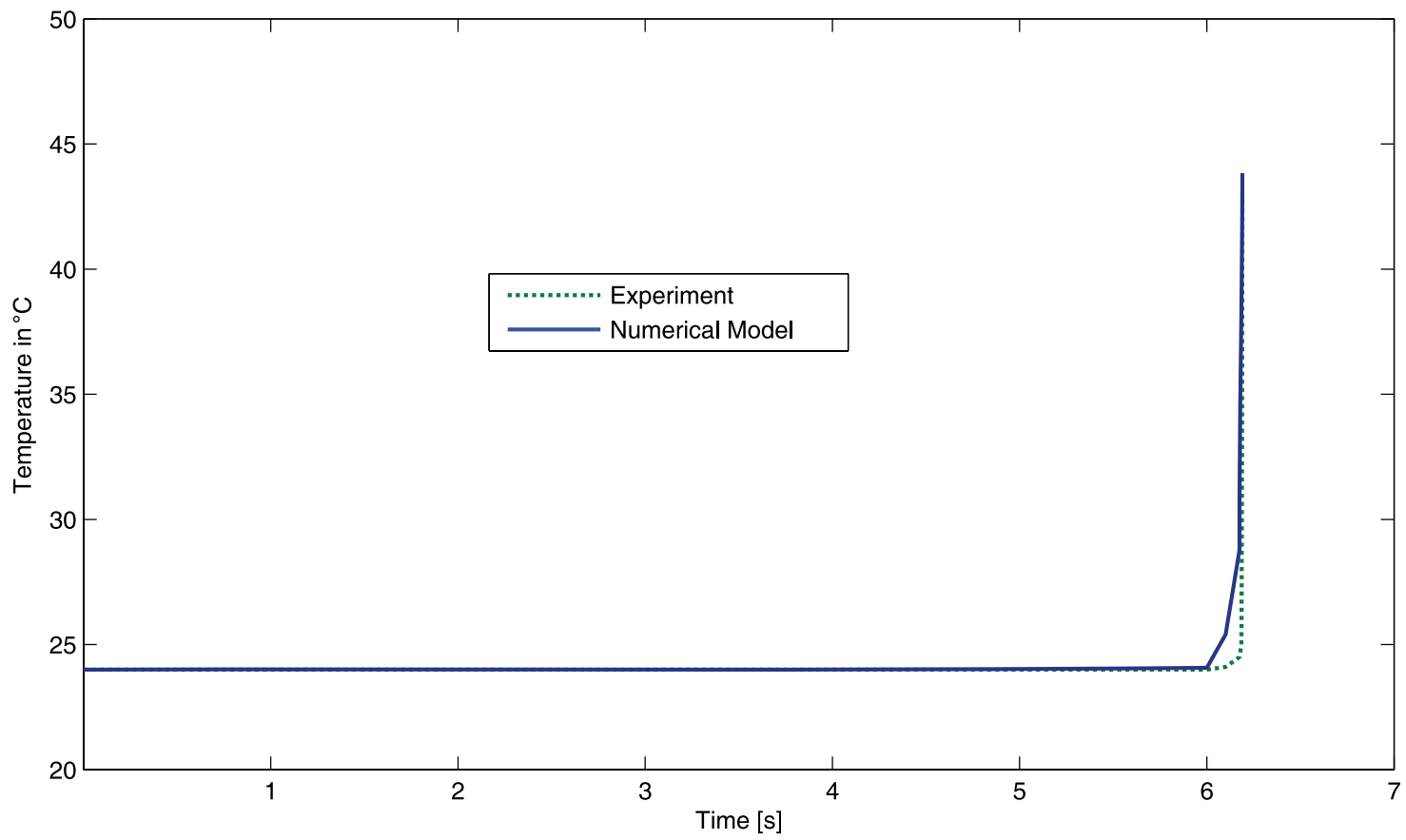

Fig. 9 Test 2: Comparison between the modeled and the experimental temperature during the formation of the fish-eye (inclusion $13.83 \mu \mathrm{m}$, eccentricity 0.83 ). 


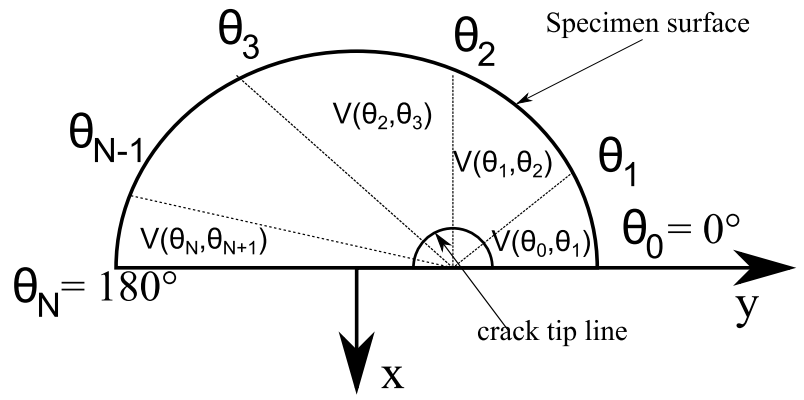

Fig. 10 Division of the crack tip line in the crack plane.

propagation. It can be seen in Fig. 12 that the temperature increase is distributed regularly around the crack tip, but is very small. The temperature in the specimen begins to increase when the crack front approaches the edge of the specimen and the stress state is not anymore the stress state of an internal crack in an infinite medium (see Figs 13 and 14).

\section{CONCLUSION}

This study has proposed a thermomechanical modelling of the propagation of a fatigue crack in VHCF fatigue regime. The plastic dissipation per cycle has been computed from 3D finite element models of stationary crack in a cylinder under constant amplitude, mode I loading. The temperature rise during fatigue crack propagation is deduced from this plastic dissipation by the resolution of a transient heat conduction problem with a moving source. The numerical results are in a good agreement with experimental test, and show that the plastic dissipation, and hence the thermal source, is concentrated near the surface of the specimen.

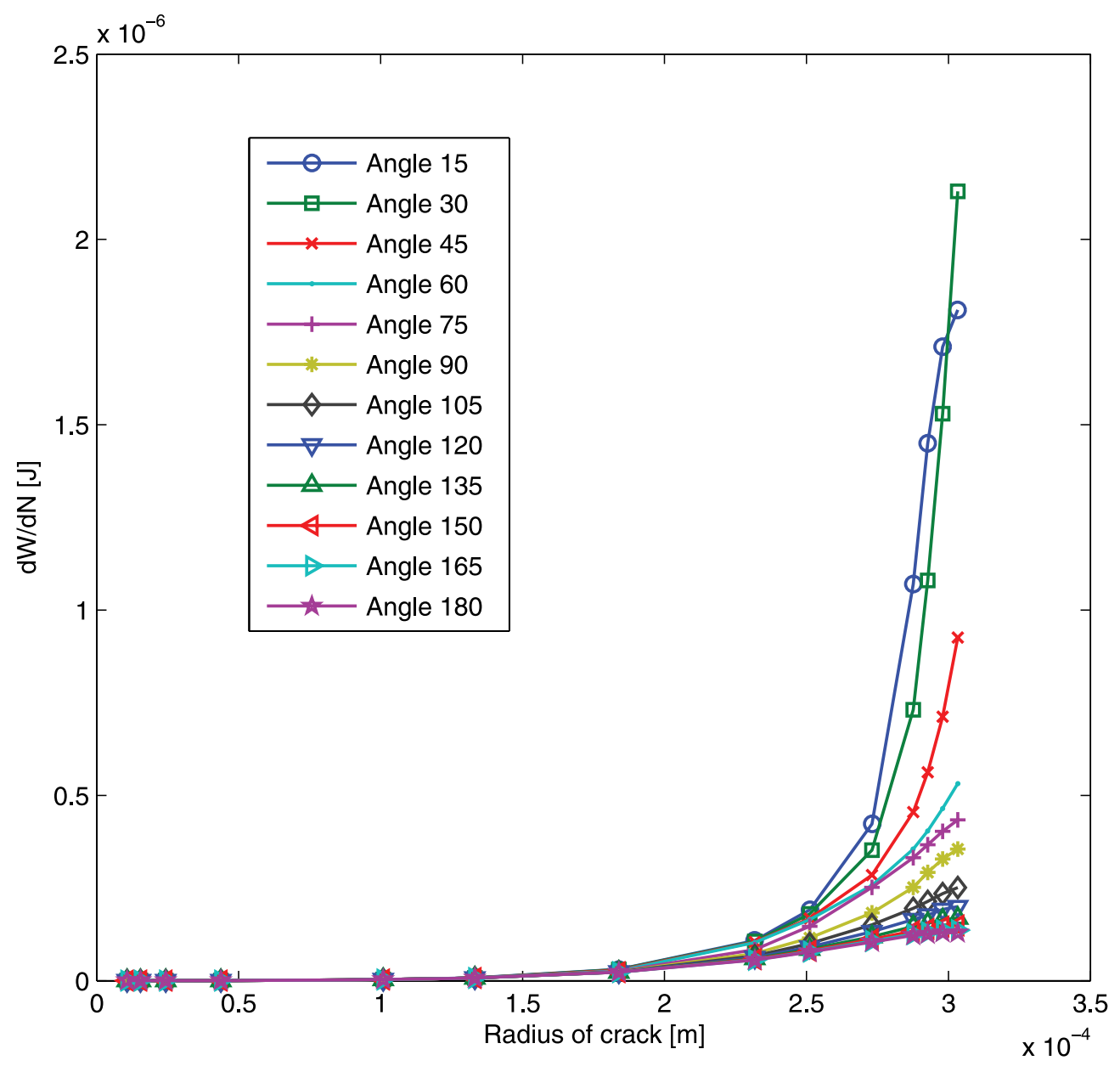

Fig. 11 Evolution of the plastic dissipation as a function of the fish-eye radius. 


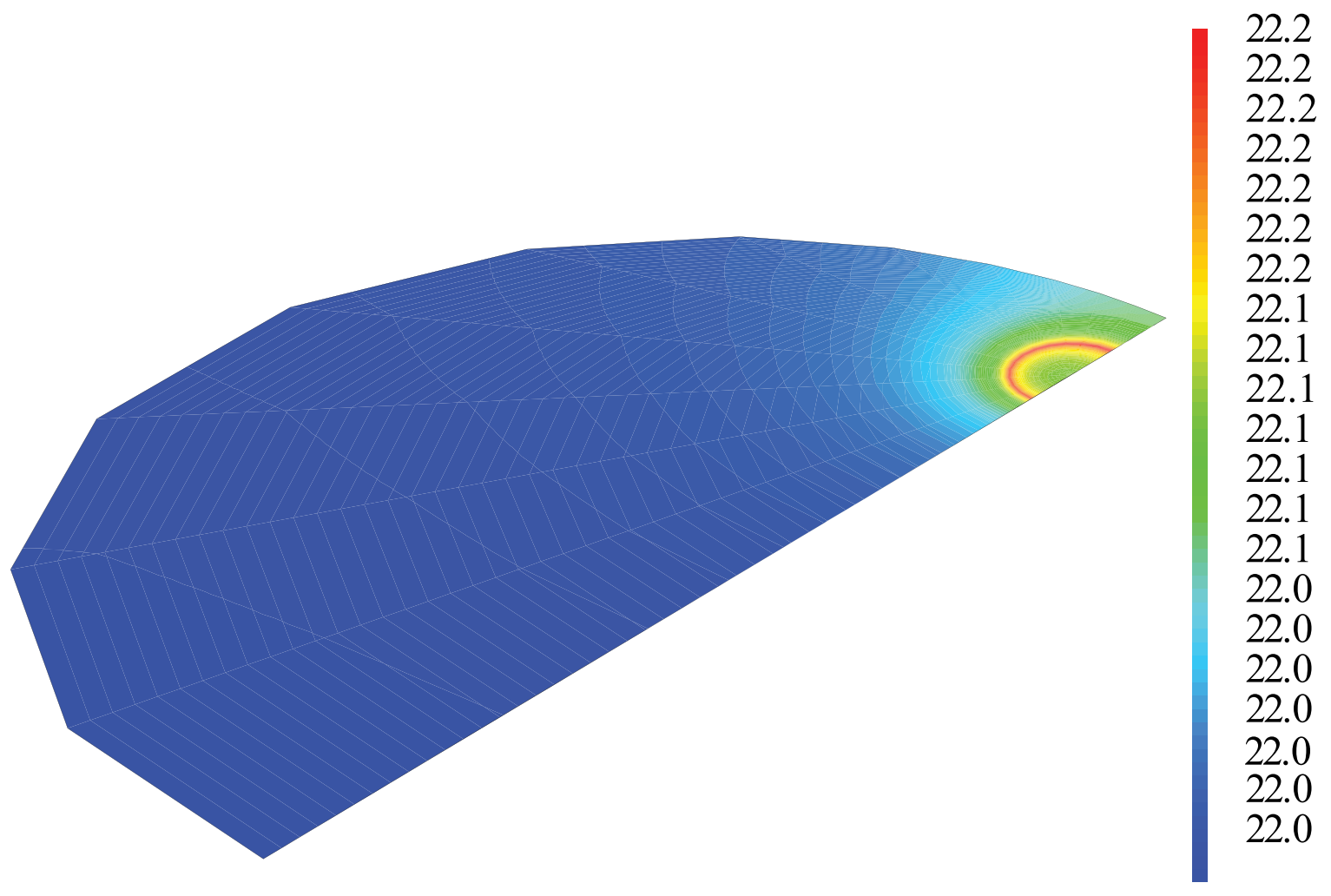

Fig. 12 Temperature field in ${ }^{\circ} \mathrm{C}$ at $t=4.5 \mathrm{~s}$.

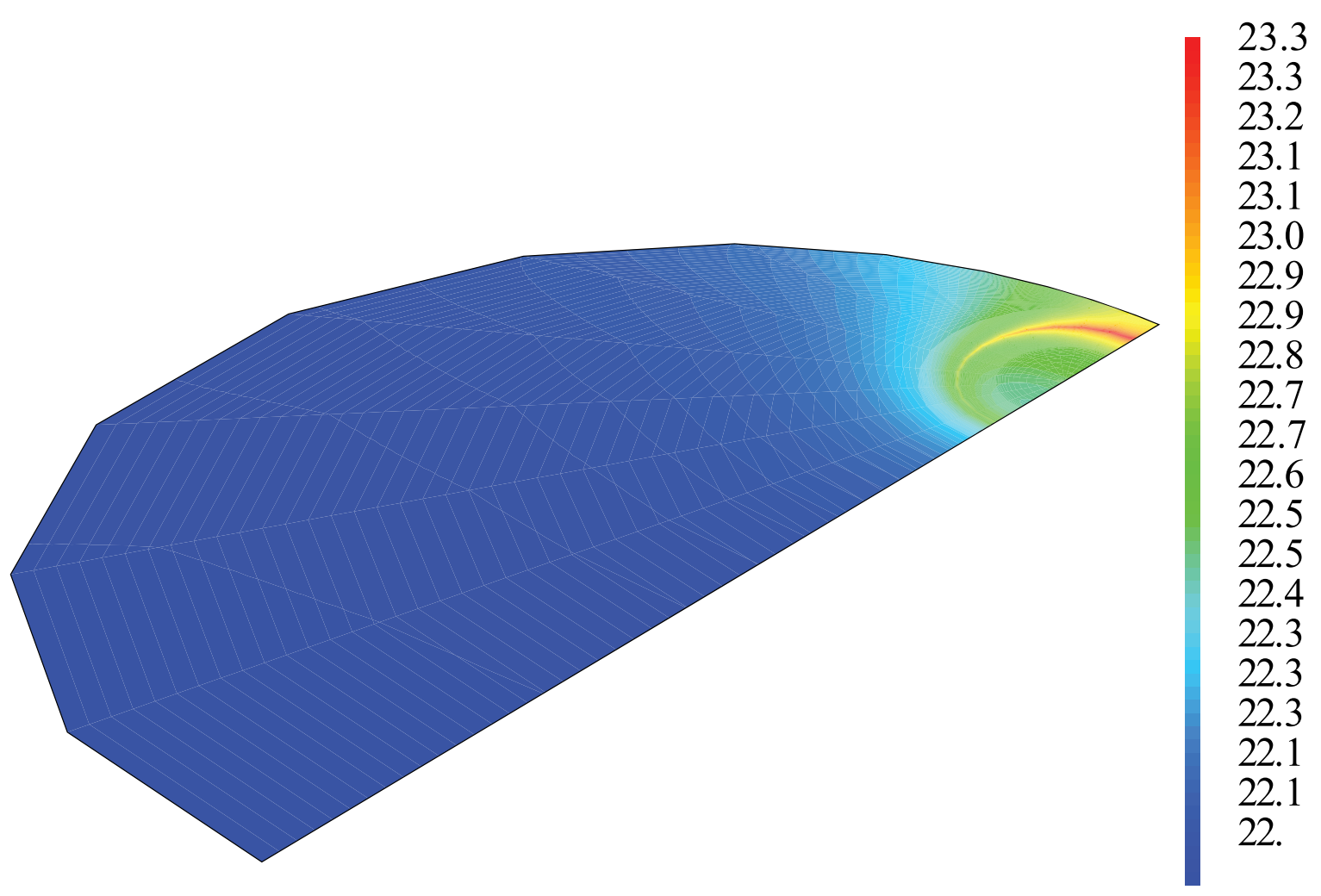

Fig. 13 Temperature field in ${ }^{\circ} \mathrm{C}$ at $t=4.71 \mathrm{~s}$. 


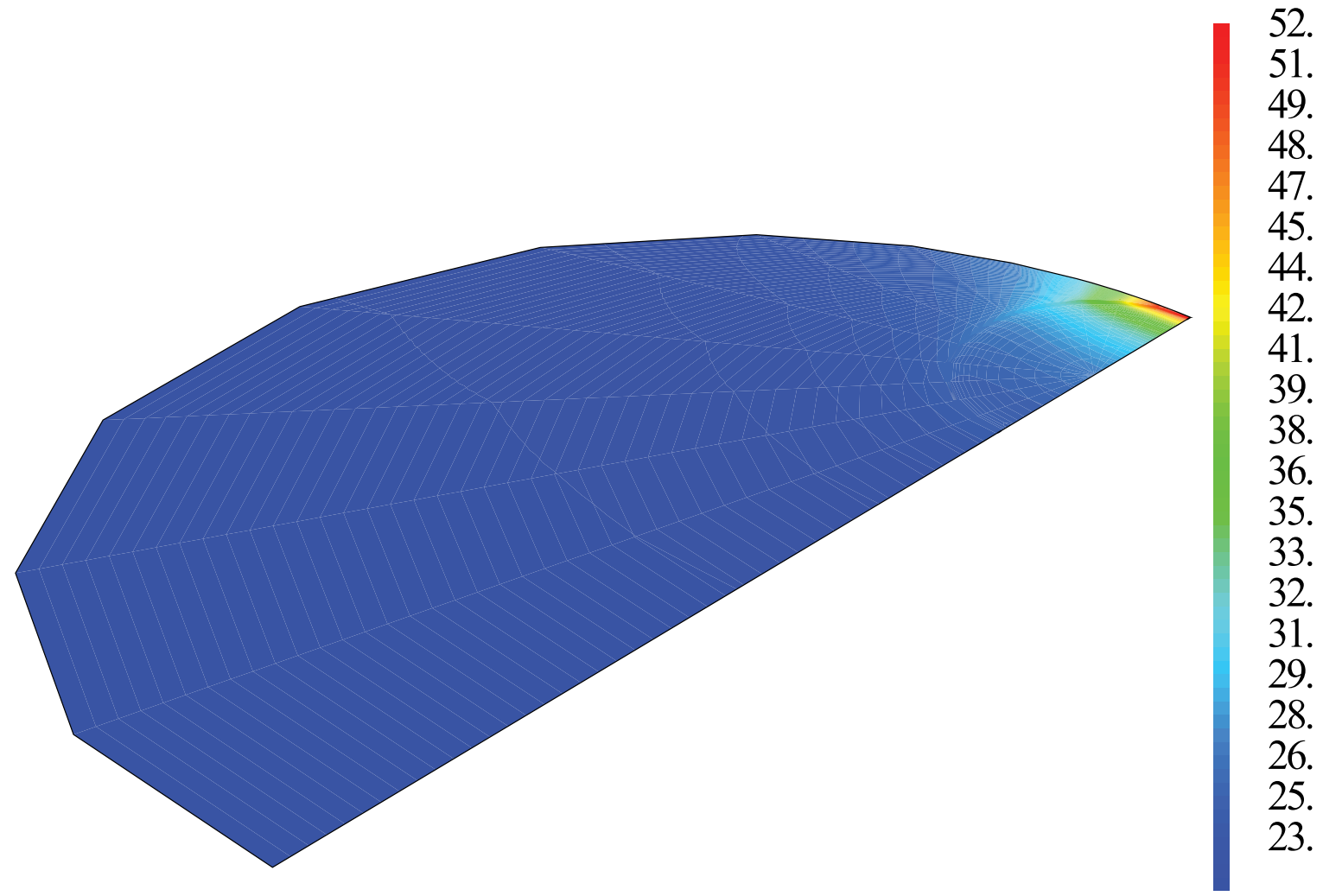

Fig. 14 Temperature field in ${ }^{\circ} \mathrm{C}$ at $t=4.84 \mathrm{~s}$.

\section{Acknowledgement}

The authors would like to express their gratitude to Dr. Zhi Yong Huang (Sichuan University, China), for the experimental results used to assess the numerical model.

\section{REFERENCES}

1 Bathias, C., Drouillac, L. and Lefrançois, P. (2001) How and why the fatigue sn curve does not approach a horizontal asymptote. Int. F. Fatigue 23, 143-151.

2 Sohar, C., Betzwar-Kotas, A., Gierl, C., Weiss, B. and Dannineg, H. (2008) Gigacycle fatigue behavior of a high chromium alloyed cold work tool steel. Int. 7. Fatigue 30, 1137-1149.

3 Li, W., Sakai, T., Li, Q., Lu, L. T. and Wang, P. (2011) Effect of loading type on fatigue properties of high strength bearing steel in very high cycle regime. Mater. Sci. Eng.: A 528, 5044-5052.

4 Mason, W. P. (1956) Internal friction and fatigue in metals at large strain amplitudes. F. Acoust. Soc. Am. 28, 1207-1218.

5 Bathias, C. and Paris, P. C. (2005) Gigacycle Fatigue in Mechanical Practice. CRC Dekker, New York

6 LaRosa, G. and Risitano, A. (2000) Thermographic methodology for rapid determination of the fatigue limit of materials and mechanical components. Int. F. Fatigue 22, $65-73$.
7 Fargione, G., Geraci, G., LaRosa, A. and Risitano, A. (2002) Rapid determination of the fatigue curve by the thermographic method. Int. F. Fatigue 24, 11-19.

8 Ranc, N., Wagner, D. and Paris, P. C. (2008) Study of thermal effects associated with crack propagation during very high cycle fatigue tests. Acta Mater. 56, 4012-4021.

9 Ranc, N., Palin-Luc, T. and Paris, P. C. (2011) Thermal effect of plastic dissipation at the crack tip on the stress intensity factor under cyclic loading. Eng. Fract. Mech. 78, 961-972.

10 Hertzberg, R. W. (1996) Deformation and Fracture Mechanics of Engineering Materials. 4th edn, John Wiley and Sons, New York.

11 Tada, H., Paris, P. C. and Irwin, G. R. (2000) The Stress Analysis of Crack Handbook. 3rd edn, ASME, New York.

12 Morabito, A. E., Chrysochoos, A., Dattoma, ASME V. and Galietti, U. (2007) Analysis of heat sources accompanying the fatigue of 2024 t3 aluminium alloys. Int. F. Fatigue 29, 977-984.

13 Taylor, G. and Quinney, H. (1937) The latent heat remaining in a metal after cold work. Proc. Roy. Soc. A163, 157181.

14 Simo, J. and Miehe, C. (1992) Associative coupled thermoplasticity at finite strains: formulation, numerical analysis and implementation. Comp. Methods Appl. Mech. Eng. 98, 41-104.

15 Klingbeil, N. W. (2003) A total dissipated energy theory of fatigue crack growth in ductile solids. Int. 7. Fatigue 25, 117-128. 
16 Marines-Garcia, I., Paris, P. C., Tada, H., Bathias, C. and Lados, D. (2008) Fatigue crack growth from small to large cracks on very high cycle fatigue with fish-eye failures. Eng. Fract. Mech. 75, 1657-1665.

17 CAST3M. see http: www-cast3m.cea.fr.

18 Ellyin, F. and Ozah, F. (2010) 3d modelling of plasticity induced fatigue crack closure-effect of materials constitutive relations. Eng. Fract. Mech. 77, 1693-1707.

19 Wu, K., Ni, J. and Bathias, C. (1994) An automatic ultrasonic fatigue testing system for studying low crack growth at room and high temperatures. ASTM Spec. Tech. Publ. 1231, 598-607.

20 Bathias, C. (2006) Piezoelectric fatigue testing machines and devices. Int. F. Fatigue 28, 1438-1445.

21 Paris, P. C., Marines-Garcia, I., Hertzberg, R. W. and Donald, J. K. (2004) The relationship of effective stress intensity, elastic modulus and burger's vector on fatigue crack growth as associated with fish-eye gigacycle phenomena. In: Proceedings of the Third International Conference on Very High-Cycle Fatigue, Kyoto. 